\title{
The interface between HOPG and 1-butyl-3-methyl-imidazolium hexafluorophosphate
}

\author{
C. Müller, ${ }^{a, b}$ K. Németh, ${ }^{c} S$. Vesztergom, ${ }^{d}$ T. Pajkossy ${ }^{e}$ and T. Jacob ${ }^{a, f}$
}

\begin{abstract}
The interface between highly oriented pyrolytic graphite (HOPG) and 1-butyl-3-metyl-imidazolium hexafluorophosphate $\left(\mathrm{BMIPF}_{6}\right)$ has been studied using cyclic voltammetry, electrochemical impedance spectroscopy, immersion charge measurements and in-situ scanning tunneling microscopy (insitu STM). The results are compared with those obtained with $A u(100)$ in $\mathrm{BMIPF}_{6}$ (Phys.Chem.Chem.Phys., 2011, 13, 11627). The main result is that the high frequency capacitance spectra on the two systems are similar to each other, however at low frequencies some slow interfacial processes cause the appearance of a second capacitance arc on $\mathrm{Au}(100)$, which is absent for HOPG. The slow processes are attributed to the rearrangement of the Au surface structure and to the formation of ionic liquid adlayers - these are visualized by in-situ STM.
\end{abstract}

\section{Introduction}

Ionic liquids (ILs) 1,2,3,4 are organic salts which are liquid (molten) at ambient temperatures. They are often referred to as "designer solvents": due to the large variety of the different possible cation/anion combinations, the physicochemical properties of ILs can be fine-tuned in order to meet the requirements of specific applications. ILs present a versatile alternative to more common solvents because of their broad (sometimes up to 4-5 V wide) electrochemical window and high thermal stability. They are non-flammable, exhibit virtually zero vapour pressure and many chemical species have a good solubility in them. From an electrochemical point of view, their most promising field of application is the design of energy-storage devices such as batteries and supercapacitors. ${ }^{5,6,7}$ In addition, ionic liquids (especially the air- and water-stable imidazolium-based ones ${ }^{8}$ ) can also be used as electrolytic media for the electrodeposition of highly reactive materials $\mathrm{s}^{9,10}$. In order to facilitate these applications, a general understanding of the physical chemistry of metal | IL in-

a Corresponding author. E-mail: claus.mueller@uni-ulm.de.

b Institute of Electrochemistry, Ulm University, Albert-Einstein-Allee 47, Ulm D-89069 Germany.

c Institute for Solid State Physics and Optics, Wigner Research Centre for Physics, Hungarian Academy of Sciences, Konkoly Thege Miklós út 29-33, H-1121 Budapest, Hungary.

d Eötvös Loránd University, Pázmány Péter sétány 1/A, H-1117 Budapest, Hungary.

e Institute of Materials and Environmental Chemistry, Research Centre for Natural Sciences, Hungarian Academy of Sciences, Magyar tudósok körútja 2, H-1117 Budapest, Hungary

f Helmholtz-Institute Ulm, Helmholtzstrasse 11, D-89069 UIm, Germany 
terfaces is required; this is the reason why ionic liquids are in the focus of a continuously growing interest in both experimental and theoretical research. ${ }^{11,12,13}$

From the fundamental research point of view, the key point of studying metal | IL interfaces is the use of electrodes with well-defined surface structures in combination with high-purity ILs. In accordance with this statement, the interface of gold single crystals with a well-defined surface orientation was recently studied in 1-butyl-3-methyl-imidazolium hexafluorophosphate (BMIPF 6 ); ${ }^{14,15,16}$ in a home-made guanidinium-based $\mathrm{IL}^{17}$ and in 1-butyl-3-methyl-imidazolium bis(trifluoromethylsulfonyl)imide $\left(\mathrm{BMITf} \mathrm{f}_{2} \mathrm{~N}\right) \cdot{ }^{18}$ In these studies the Faradaic reaction-free potential regions of the metal | IL electrodes were identified and characterized by cyclic voltammetry (CV), while electrochemical impedance spectroscopy (EIS) was employed in order to determine the interfacial capacitance. The potential-dependent capacitance spectra, as well as the surface images acquired by scanning tunnelling microscopy (in-situ STM) allowed for the characterization of the interfacial structure and of the different processes occurring therein. By means of immersion charge measurements, the potential of zero total charge (pztc) of metal | IL interfaces was also determined, providing further information on the chemical nature of the interfacial adlayers.

Interestingly, the results of our previous studies ${ }^{14-18}$ contradicted in one crucial point the existing theories of metal | IL interfaces. Kornyshev's original theory ${ }^{19}$ and some related works ${ }^{20,21,22,23,24}$ (see also Ref. 25 for a review) attempt to describe ionic liquid systems on the basis of a lattice gas model by taking into consideration Coulomb interactions as well as the finite volume of the ionic species. These models ${ }^{19,20,21,22}$ predict bell- or camel-shaped differential capacitance vs. electrode potential curves, however - as it was also pointed out by Kornyshev et al. in a recent study ${ }^{23}-$, the numerical values significantly overestimate the experimentally determined capacitance data. This discrepancy may be resolved by assuming a lower-than-unity ionic dissociation factor in ILs: ${ }^{23,26,27,28,29,30}$ as the mean-field capacitance in Kornyshev's model depends linearly on the ionic concentration, a lower ionic concentration could, in principle, bring the results of theory and experiment closer to each other.

However, one contradiction still remains to be dealt with: namely, that time does not appear as a variable in the existing double-layer theories of IL systems, and as a consequence, the theoretically predicted $C(E)$ functions are always frequency-independent. This is in strong contrast to the results of our previous impedance studies, which explicitly pointed out that the measured capacitance heavily depends on the frequency used for its determination. For example, it was shown that the complex plane capacitance spectra of the $\mathrm{Au}(100) \mid \mathrm{BMIPF}_{6}$ interface exhibit a characteristic "double-arc" feature. ${ }^{15} \mathrm{As}$ it is shown in this paper, explaining the double-arc behaviour of the $\mathrm{Au}(100) \mid \mathrm{BMIPF}_{6}$ system is possible by means of a comparison to the capacitance spectra recorded on highly oriented pyrolytic graphite (HOPG) | $\mathrm{BMIPF}_{6}$.

In the present work we thus present a basic electrochemical characterization of the interface of HOPG in $\mathrm{BMIPF}_{6}$, which is then compared to those results obtained with $\mathrm{Au}(100)$ in the same IL. ${ }^{15,16}$ The focus is on the following questions: $i$.) What is the physical background of the double-arc on the capacitance spectrum? ii.) Which processes could be responsible for the appearance of the two different time constants? iii.) In general, how to measure the double layer capacitance of interfaces involving ILs? 


\section{Experimental methods}

\section{Materials}

The experiments were carried out with a HOPG sample of square shape $(12 \mathrm{~mm} \times 12 \mathrm{~mm}$, Veeco, USA). Prior to each measurement, the HOPG surface was prepared by mechanical exfoliation (i. e., the topmost graphite layers were removed by scotch-tape). ${ }^{31}$ BMIPF $_{6}$ was purchased from Merck $\mathrm{KGaA}$ in the highest available quality (purity $\geq 99.5 \%$, water $\leq 100 \mathrm{ppm}$, halides $\leq 100 \mathrm{ppm}$ ). The ionic liquid was vacuum-dried for 24 hours at $80{ }^{\circ} \mathrm{C}$ and purified with a $0.3 \mathrm{~nm}$ molecular sieve. ${ }^{32}$ The electrochemical measurements were carried out in a glovebox (MBraun LabStar) under $\mathrm{N}_{2}$ atmosphere, and a protective $\mathrm{N}_{2}$ blanket was maintained over the cell also during the in-situ STM studies.

\section{Cells and basic measurements}

CV and EIS experiments were conducted using an IM6 potentiostat (Zahner Messsysteme) in a custom-made, cylindrical, closed Kel- $\mathrm{F}^{\mathrm{TM}}$ cell with a total volume of $0.3 \mathrm{~cm}^{3}$. The HOPG working electrode (WE) and the polycrystalline gold sheet counter electrode (CE) comprised the two end-faces of the cylinder. Their parallel position ensured a strictly homogeneous current distribution, which is a necessary condition of accurate high-frequency impedance measurements. A silver wire of 0.5 $\mathrm{mm}$ diameter covered by $\mathrm{AgCl}$ was applied as a quasi-reference electrode (RE), placed in-between the working and counter electrodes, parallel to both. Unless it is stated otherwise, the electrode potential values reported in this paper are all meant vs. this reference electrode. Test measurements with dummy cells were performed in order to determine phase shifts due to the potentiostat and cables.

For studying temperature dependences, a heatable copper block was mounted on the cell. A temperature control with an accuracy of $\pm 1{ }^{\circ} \mathrm{C}$ was achieved, with an accessible range of $25-130{ }^{\circ} \mathrm{C}$.

For the determination of pztc, a Kel- $\mathrm{F}^{\mathrm{TM}}$ cell comprising two parts were designed. The bottom part held the HOPG sample to which a $3 \mathrm{~mm}$ high ring was assembled in order to form a vessel. The upper part was a tube, in the wall of which a Pt wire and an $\mathrm{AgCl}$-coated $\mathrm{Ag}$ wire were inserted (these were used as counter and reference electrodes, respectively). By using a safety pipette ball (Peleus ball) mounted on the top end, the tube was filled through its porous lower end with BMIPF 6 . After a certain time, the tube was positioned over the HOPG surface (at an approximate distance of $2 \mathrm{~mm}$ ), the potential control was switched on, and the cell was flood with the electrolyte by exerting pressure on the Peleus ball. The wetting of HOPG took less than 1 second, and the current involved in the creation of the electrochemical double layer was measured. Note that using this setup we could not achieve the level of cleanliness and time resolution of the setup dedicated for immersing $\mathrm{Au}(100)$ into $\mathrm{BMIPF}_{6}{ }^{33}$

\section{Impedance measurements and spectrum analysis}

The EIS measurements (recording of spectra at different potential setpoints) were carried out by giving a special attention to the reproducibility of the results. First a spectrum was recorded at an electrode potential close to the open circuit value, between $100 \mathrm{mHz}$ and $1 \mathrm{MHz}$ (sinusoidal perturbation with $5 \mathrm{mV}$ amplitude). Several hours later, the same measurement was repeated, and the resulting spectra were compared. If the impedance plots featured no significant differences, spectra were also measured at other potential setpoints. The sequence of impedance measurements was started at $400 \mathrm{mV}$, and the setpoint values were decreased in $200 \mathrm{mV}$ steps to $-400 \mathrm{mV}$. The direction of potential stepping was changed at this point: the next applied setpoint was of $-300 \mathrm{mV}$, and then the potential was gradually stepped back to $300 \mathrm{mV}$ (again in $200 \mathrm{mV}$ steps). Thus, imped- 
ance spectra over the voltage range between 400 and $-400 \mathrm{mV}$ were recorded (at a resolution of $100 \mathrm{mV}$ ), and the cyclic sequence definition provided additional means to check the reproducibility of the spectra. (In a stable system, the shape of an impedance spectrum should depend only on the actual potential, and not on the potential at which the electrode was previously conditioned.) The invariance of the spectra for the Kramers-Kronig transformation ${ }^{34}$ was also checked in each case, and the low frequency data points for which the invariance did not hold, were removed.

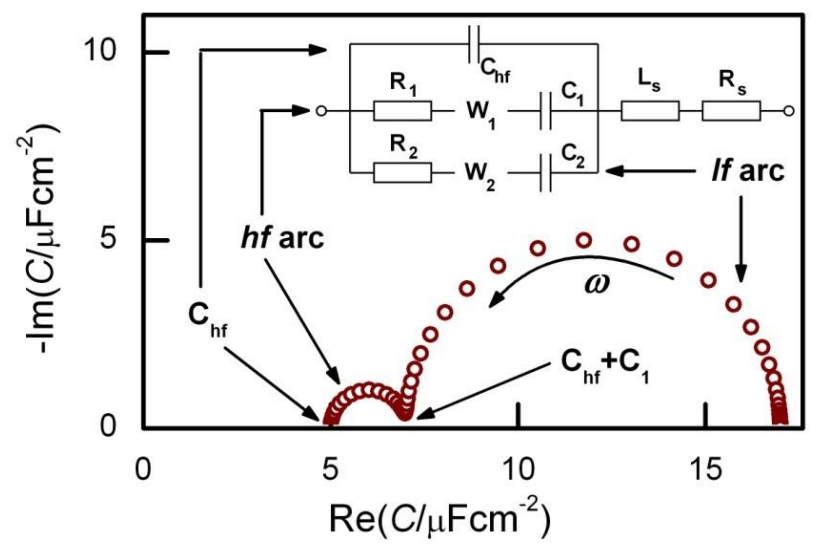

Fig. 1 Equivalent circuit used for fitting the spectra and features of the capacitance spectra of the interface. If and $h f$ indicate high and low frequency, respectively.

The measured spectra were analyzed by fitting the parameters of an equivalent circuit using modulus weighting. ${ }^{35}$ In our previous studies ${ }^{14,15,16,17,18}$ we found that the impedance spectra of most metal | IL interfaces can well be fitted by an equivalent circuit shown in the inset of Fig. 1 . Here the three-branched part represents the interfacial impedance; $R_{\mathrm{s}}$ denotes the electrolyte resistance, and an empirical, formally inductive $L_{s}$ term (of either sign, with a corresponding impedance of $i \omega L_{s}$ ) accounts for the minor high frequency errors which occur due to the various imperfections of the measurement setup. ${ }^{36}$ (Here $\omega=2 \pi f$ is the angular frequency, and $f$ the frequency of the sinusoidal perturbation.) In many cases the $\mathrm{R}_{2} \mathrm{~W}_{2} \mathrm{C}_{2}$ branch of the interfacial impedance is either missing, or some of its elements may be quasi short-circuits. The $W_{1}$ and $W_{2}$ elements are empirical terms, their pseudo-Warburg-impedance name comes from the $\sqrt{i \omega}$ frequency dependence involved. In most cases the impedance spectra - measured over 5-7 orders of magnitude of frequency - could be well fitted with 4-7 parameters of the complete circuit of Fig. 1.

In Fig. 1, and also later in this paper we visualize impedances as capacitance spectra of the interface, $C(\omega)$. This is calculated from the interfacial admittance $Y_{i}(\omega)$ as $C(\omega)=Y_{i}(\omega) /(\mathrm{i} \omega)$. Since both $R_{\mathrm{s}}$ and $L_{\mathrm{s}}$ affect impedance spectra at the high frequency end, $C(\omega)$ can be calculated as

$$
C(\omega)=1 /[\mathrm{i} \omega(\mathrm{Z}(\omega)-Z(\omega \rightarrow \infty))]=1 /\left[\mathrm{i} \omega\left(Z(\omega)-R_{\mathrm{s}}-\mathrm{i} \omega L_{\mathrm{s}}\right)\right]
$$

In the case of a circuit of Fig. 1, the $\mathrm{C}(\omega)$ function, plotted on the complex plane (as an Arganddiagram or Nyquist-plot) consists of two arcs as illustrated there.

\section{Single-frequency capacitance scans}

$\mathrm{C}(\mathrm{E})$ curves were measured by the application of single sinusoid perturbations (with $5 \mathrm{mV}$ amplitude and $1 \mathrm{kHz}$ frequency) superimposed on a stair-case-like voltage scan. Before each scan, the $R_{s}$ 
and $L_{s}$ terms of the bulk impedance (see Fig. 1) were determined by recording a single impedance spectrum, and by carrying out equivalent circuit fitting for the high-frequency parts. The impedance values were then converted (Eq. (1)) to complex capacitances, $C(E)$. Double layer capacitance is identified as the real part of $C(E)$, provided that the imaginary part is negligibly small.

\section{STM measurements}

The STM measurements were carried out on a Topometrix Discoverer TMX 2010 instrument. The STM tips were prepared by the galvanic etching of platinum/iridium wires (80/20) in $3.5 \mathrm{M} \mathrm{NaCN}$. In order to reduce the Faradaic current and the stray capacitance of the tip, a BASF electrophoretic paint was applied to the tip, except for its very end. The STM cell had a volume of $0.2 \mathrm{~cm}^{3}$ and was open to enable a 4 electrode configuration. $\mathrm{An} \mathrm{Ag} / \mathrm{AgCl}$ wire was acting as a quasi-reference electrode and a Pt wire was used as a counter electrode. Constant current scanning with a typical setpoint of $1 \mathrm{nA}$ was carried out. The cell was filled with the IL inside a glove-box, and was transferred to the STM in a small chamber providing $\mathrm{N}_{2}$ protection.

\section{Results}

\section{Cyclic voltammetry}

The electrochemical stability window of HOPG $\mid$ BMIPF $_{6}$ was identified by cyclic voltammetry, as shown in Fig. 2(a). The CV in the narrowest potential range (measured between $\pm 400 \mathrm{mV}$ ) exhibited featureless capacitive behavior, although the CV was not perfectly symmetrical to the horizontal axis. After broadening the scanned range to $-0.6 \mathrm{~V}$ in the negative and $+1.5 \mathrm{~V}$ in the positive direction, the system still remained fairly stable, and the only notable change was the appearance of a pair of spikes at $+1.2 \mathrm{~V}$ (see also the results of in-situ STM studies below). With further extension of the potential vertices, high negative and positive Faradaic currents were triggered below $-2.8 \mathrm{~V}$ and above $+2.0 \mathrm{~V}$, respectively - hence the "electrochemical window" is about $4.8 \mathrm{~V}$ broad.

The charging current measured on HOPG | $\mathrm{BMIPF}_{6}$ is lower by a factor of 3 to 5 compared to that measured on $\mathrm{Au}(100) \mid \mathrm{BMIPF}_{6}{ }^{16}$ and the same applies for the $d c$ capacitance - defined as the average charging current divided by the scan rate -, that has an approximate value of $7 \mu \mathrm{F} / \mathrm{cm}^{2}$.

The cyclic voltammograms taken between -0.4 and $+0.4 \mathrm{~V}$ remain stable, even following a longtime conditioning at a certain potential. As an example, Fig. 2(b) shows CVs, which were recorded before and after an impedance measurement that took three hours at $E=0 \mathrm{~V}$ : the charging current is only slightly increased, and the small Faradaic currents appearing at positive potentials are also negligible.

\section{Impedance measurements}

At first sight the impedance spectra measured between -0.4 and $+0.4 \mathrm{~V}$ are capacitive. A closer inspection of the Nyquist representation of the interfacial capacitance (calculated according to Eq. (1)) reveals, however, a single-arc shape (see Figs. 3(a), 4 and 6(a)). Accordingly, the equivalent circuit of the interface is basically two-branched (upper inset of Fig. 3(a), the $R_{2}$ element adds a little correction only). The quality of the fits is good, as it is illustrated by the residuals plotted in Fig. 3(b). In general, the spectra obtained from HOPG | BMIPF $_{6}$ can be fitted with the same accuracy as those 

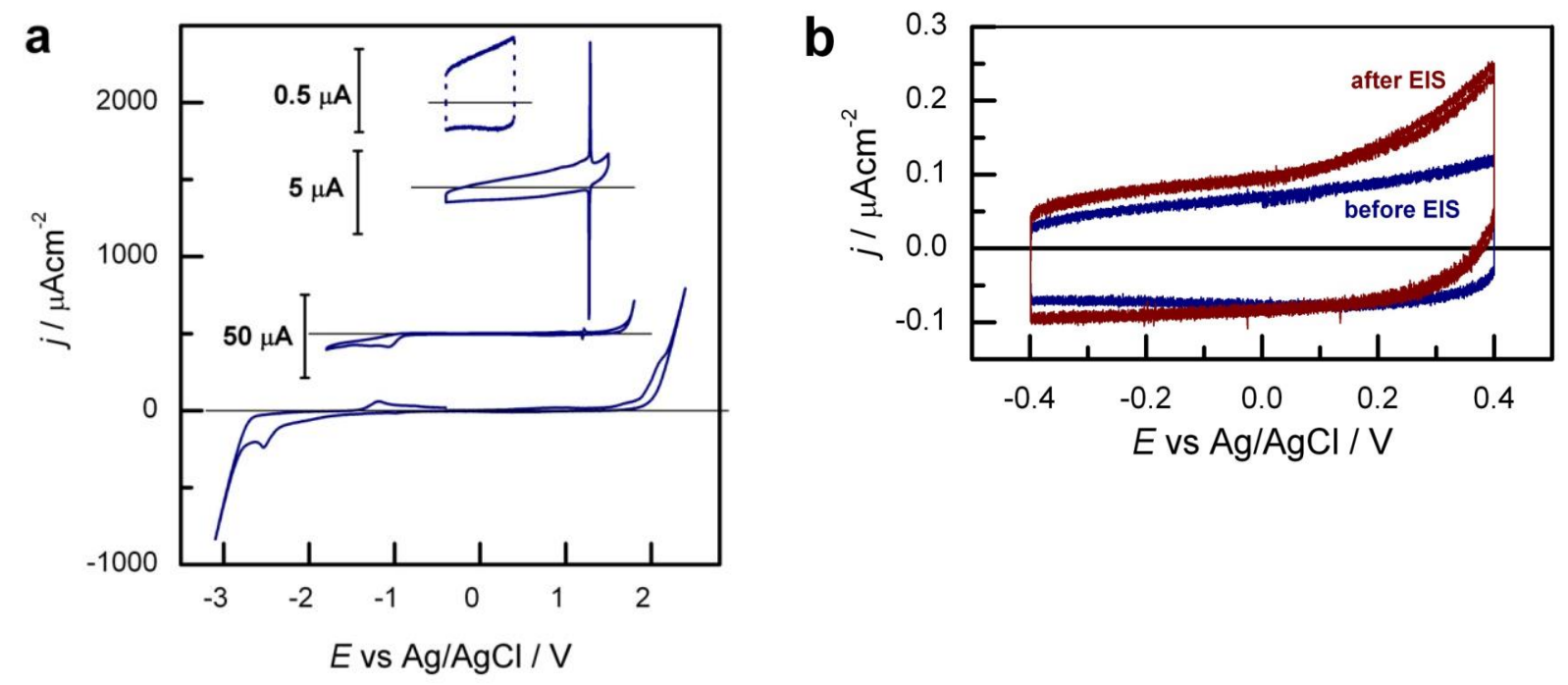

Fig. 2 (a) CVs at $50 \mathrm{mV} / \mathrm{s}$ with varied potential limits. For the sake of visibility, the CVs are shifted along the ordinate and magnified as indicated. (b) CVs recorded at $10 \mathrm{mV} / \mathrm{s}$ before and after an EIS measurement (lasting for 3 hours) was carried out at $0 \mathrm{~V}$ vs. $\mathrm{Ag} \mid \mathrm{AgCl}$.

obtained from $\mathrm{Au}(100) \mid \mathrm{BMIPF}_{6} ; \chi^{2} \approx 10^{-5}$ for both cases. The fitting residuals in Fig. $3 \mathrm{~b}$ are just as little as they are in Fig. $2 b$ of Ref. 15. We note that this equivalent circuit was found appropriate also when potential and temperature dependences were studied (see the results of Figs. 4 and 6).
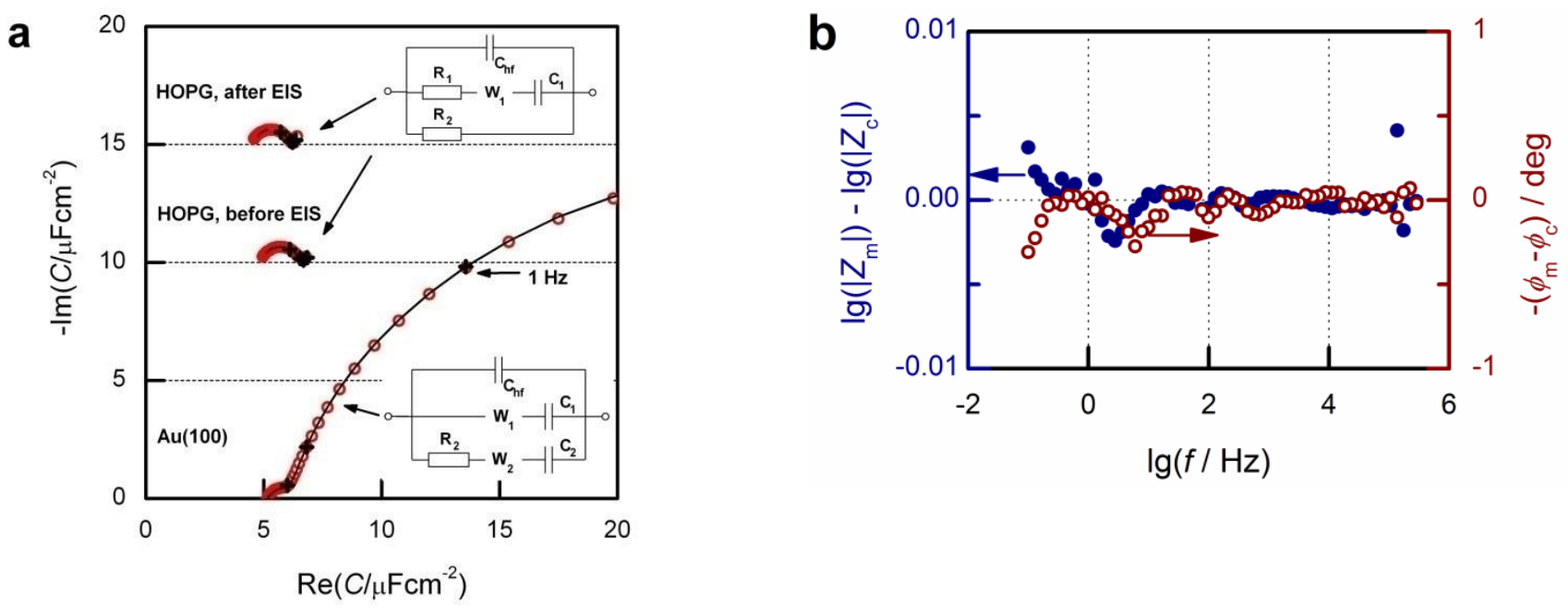

Fig. 3 (a) Complex plane plot of the interfacial capacitance spectra, in the $0.1 \mathrm{~Hz}-1 \mathrm{kHz}$ range, measured at $0 \mathrm{~V}$ on HOPG and $\mathrm{Au}(100)$. The two HOPG spectra were measured before and after the application of a potential sequence (Fig. 4), and demonstrate the high stability of the system. The $\mathrm{Au}(100)$ spectrum is taken from Fig. 5 of Ref. 16. Open symbols represent the measured spectrum points, to which the solid lines were fitted using the equivalent circuits of the insets. The points measured at $0.1,1,10$ and $100 \mathrm{~Hz}$ are marked by crosses. The spectra are shifted along the ordinate for reasons of clarity. (b) Fitting residuals for the "HOPG, before EIS series" spectrum of the (a) part of the figure. The full and open symbols are differences of impedance magnitudes and phases, respectively. 
The high frequency limit of the capacitance spectra for HOPG and $A u(100)$ are approximately the same, both having a value of $\approx 5 \mu \mathrm{F} / \mathrm{cm}^{2}$. Also the medium frequency part is similar for the two systems: the middle branch of the interfacial equivalent circuit (insets of Fig. 3a) contains a pseudoWarburg impedance $\left(W_{1}\right)$ and a capacitance $\left(C_{1}\right)$, eventually also a resistor $\left(R_{1}\right)$. The origin of the pseudo-Warburg impedance is still unclear and has been used only in an empirical manner. For "real" Warburg impedances, this element is due to the diffusion of some minority components, which can be excluded in case of our system. $\mathrm{R}_{1}$ and $\mathrm{C}_{1}$ deliver a time-constant in the order of $20 \mathrm{~ms}$.

The main difference between the capacitance spectra of HOPG and Au(100) lies in the low frequency range. The second arc on the capacitance spectrum, which is present for $A u(100)$, is missing at the same frequency range for HOPG (Fig. 3(a)). Accordingly, the impedance of the $\left(\mathrm{R}_{2} \mathrm{~W}_{2} \mathrm{C}_{2}\right)$ branch on HOPG is so high that only an $\mathrm{R}_{2}$ element of very high value (and also of high uncertainty) can be determined by fitting.

Here we note that the single-arc shape of the capacitance spectra is reproducible. The two spectra shown in Fig. 3(a), recorded before and after a long series of measurements, are practically identical; however, the actual capacitance values - similarly to what was shown by cyclic voltammetry are slightly increased following a long-time conditioning at several potentials.

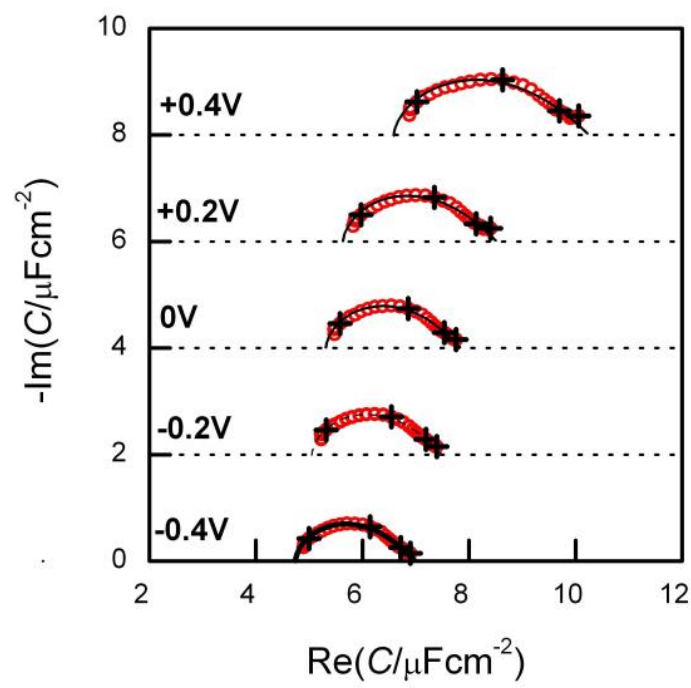

Fig. 4 Potential dependence of capacitance spectra of HOPG in $\mathrm{BMIPF}_{6}$ : measured data points (open symbols) combined with fitted ones (solid lines). Crosses are points at $1 \mathrm{kHz}, 100 \mathrm{~Hz}, 10 \mathrm{~Hz}$ and $1 \mathrm{~Hz}$ frequencies. The spectra are shifted along the ordinate for clarity.

The capacitance spectra of HOPG exhibit only a slight potential dependence in the $-0.4 \mathrm{~V}$ to $+0.4 \mathrm{~V}$ potential range, as shown in Fig. 4 . The high-frequency capacitance $\left(C_{\mathrm{hf}}\right)$ lies around $5 \mu \mathrm{F} \mathrm{cm}{ }^{-2}$, and seems to increase with positive potentials. $C_{1}$ is in the order of magnitude of $2 \mu \mathrm{F} \mathrm{cm}^{-2}$ and shows a similar potential dependence. The fitted values of $C_{\mathrm{hf}}(E)$ and $C_{\mathrm{hf}}(E)+C_{1}(E)$ are plotted for HOPG in Fig. 5, where for the sake of comparison the dataset of Fig. 5 in Ref. 16 (measured on $\mathrm{Au}(100)$ in $\mathrm{BMIPF}_{6}$ ) is also included.

Although the shape of the curves in Fig. 5 seems to be somewhat different for the two electrodes, it should be noted that in terms of numerical values these differences are rather low $\left(<1 \mu \mathrm{Fcm}{ }^{-2}\right.$ for $C_{\mathrm{hf}}(E)$, and $<2 \mu \mathrm{Fcm}^{-2}$ for $\left.C_{\mathrm{hf}}(E)+C_{1}(E)\right)$. 
The potential dependence of $C_{\mathrm{hf}}$ can be directly determined by single-frequency/scanned-potential impedance measurements as well. This overcomes the rather time-consuming recording of entire impedance spectra. As it is shown in Fig. $4,1 \mathrm{kHz}$ is a sufficiently high frequency, where $C_{\mathrm{hf}}(E) \approx \operatorname{Re}(C(E))$. Interfacial capacitances as function of potential determined at $1 \mathrm{kHz}$ are shown in Fig. $5(b)$. This curve exhibits the same increase of $C_{\mathrm{hf}}$ with potential, as obtained from the impedance spectra.

a

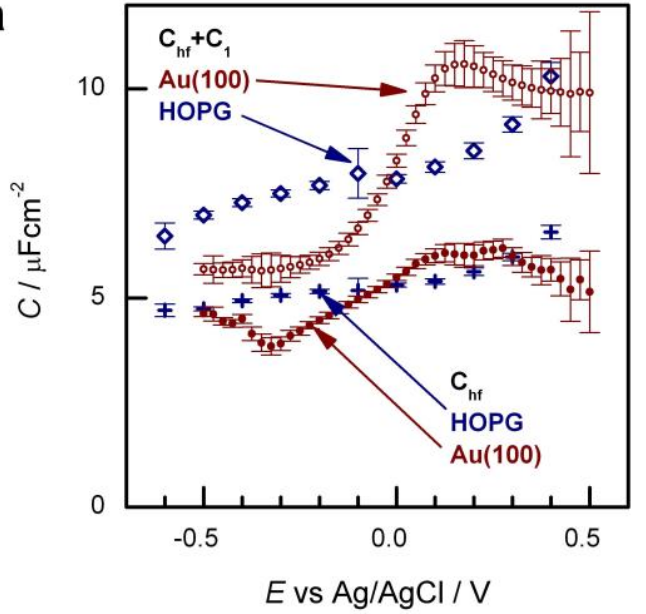

b

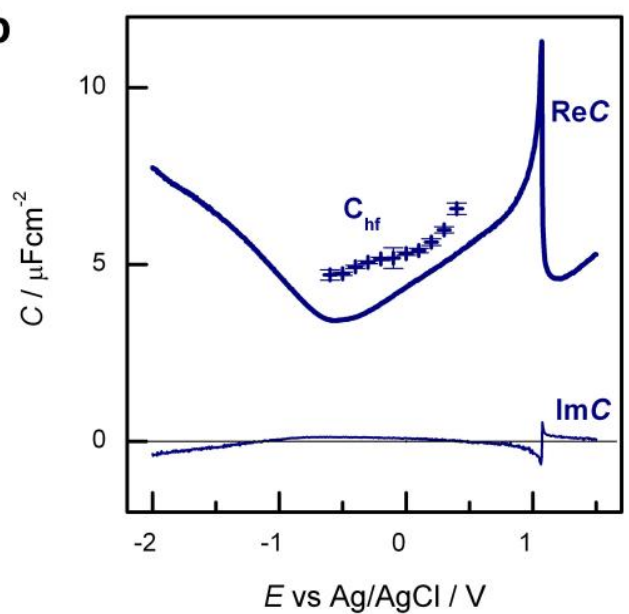

Fig. 5 (a) Fitted capacitances as a function of potential. Crosses and diamonds: $C_{\mathrm{hf}}$ and $C_{\mathrm{hf}}+C_{1}$ for HOPG, respectively. Full and open circles show the re-plotted dataset of Fig. 5 of Ref. 16 for $C_{\mathrm{hf}}$ and $C_{\mathrm{hf}}+C_{1}$ on $\mathrm{Au}(100)$, respectively. (b) Solid lines: $\operatorname{Re}(C)$ and $\operatorname{Im}(C)$ measured at $1 \mathrm{kHz}$ frequency and a $2.8 \mathrm{mV} / \mathrm{s}$ effective scan-rate on HOPG. The $C_{\text {hf }}$ dataset of (a) was re-plotted here for comparison.

a

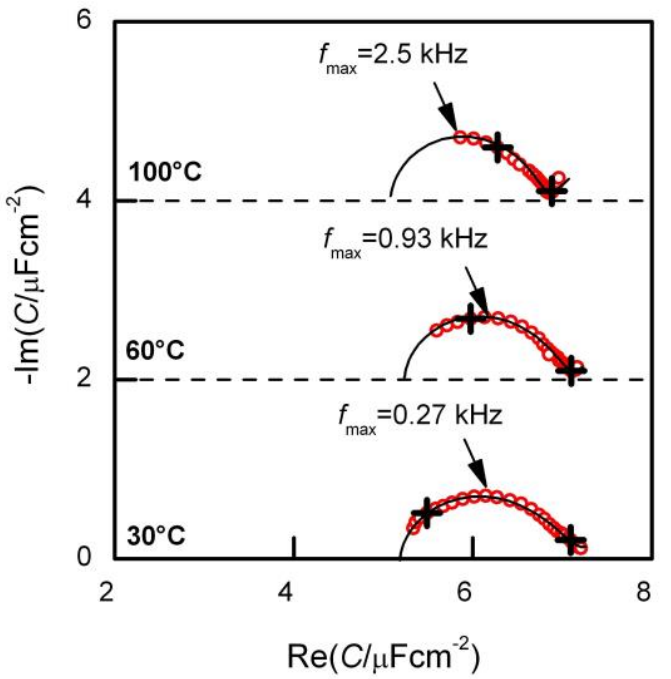

b

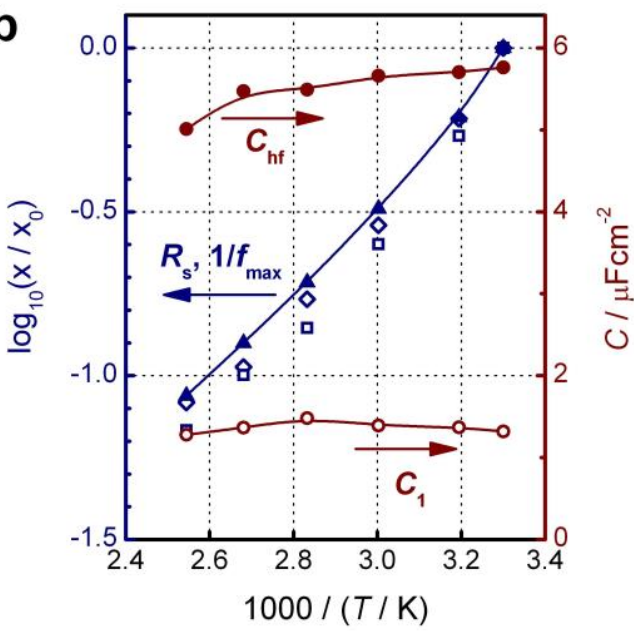

Fig. 6 (a) Capacitance spectra of HOPG in $\mathrm{BMIPF}_{6}$ at the indicated temperatures, recorded at $0 \mathrm{~V}$. Open symbols and solid lines represent measured and fitted data, respectively. Crosses are points at $1 \mathrm{kHz}$ and $10 \mathrm{~Hz}$ frequencies; $f_{\max }$, the characteristic frequency of the $\mathrm{R}_{1} \mathrm{~W}_{1} \mathrm{C}_{1}$ branch (the frequency of the arc's topmost point) is indicated. The spectra are shifted along the ordinate for clarity. (b): Fitted parameters as a function of reciprocal temperature. Left axis: $x$ represents any of $R_{\mathrm{s}}$ (full triangles), $R_{1} C_{1}$ (open diamonds) and $1 / f_{\max }$, (open squares), all normalized to their $30^{\circ} \mathrm{C}$ value, $x_{0}$. Right axis: $C_{\mathrm{hf}}$ (full circles), $C_{1}$ (open circles). 
As in the case of $\mathrm{Au}(100) \mid \mathrm{BMIPF}_{6}{ }^{16}$ we attempted to identify the origin of the equivalent circuit elements. Therefore, we measured impedance spectra at different temperatures (between room temperature and $120^{\circ} \mathrm{C}$ ). It is apparent that all the capacitance spectra in Fig. $6(\mathrm{a})$ are of single-arc shape and the high and low frequency limits have little if any temperature dependence (Fig. 6(b), dataset belonging to the right axis). We note that an increase of double layer capacitances with increasing temperature was previously reported, ${ }^{25,37}$ and the $C_{\mathrm{hf}}$ values measured on $\mathrm{Au}(100)$ | $\mathrm{BMIPF}_{6}$ (cf. Figs. 6 and 7 of Ref. 16) also show the same behaviour. However, the relative changes of $C_{\mathrm{hf}}$ are negligible compared to the temperature dependence of the resistive elements of the circuit.

$R_{\mathrm{s}}$ and the characteristic frequency (reciprocal of the time constant) of the arc do depend on temperature. The latter dependence is apparent in Fig. 6(a): the capacitance spectra points shift clockwise along the arc with increasing temperature. The time constant of the arc can be given in two simple approximations: as the reciprocal frequency of the topmost point of the arc, $f_{\max }$, or as the $R_{1} C_{1}$ product. These values are rather similar to each other and depend strongly on the temperature. Their temperature dependence is similar to that of $R_{\mathrm{s}}$, as shown in Fig. $6(\mathrm{~b})$, data linked to the left axis. Note that the slope of the $\log \left(R_{s}\right)$ vs. $1 / T$ curve is related to the activation energy - in the order of $30 \mathrm{~kJ} / \mathrm{mol}$ - of ion transport in the bulk.

\section{Immersion experiments}

We obtained the immersion charge by integrating the current following the contact of HOPG with $\mathrm{BMIPF}_{6}$ under controlled potential; pztc is the potential at which the integration charge is just zero. The integrated current transients recorded at different immersion potentials are shown in Fig. 7. With HOPG, the immersion charge is much smaller than with $\mathrm{Au}(100)$. As it is shown in the inset, the charge - calculated for the first second; i. e., the time of the wetting - is in the $1 \mu \mathrm{C} / \mathrm{cm}^{2}$ order of magnitude in the $100 \mathrm{mV}$ vicinity of the pztc. This low value is in accordance with the small capacitances $\left(C_{\mathrm{hf}}+C_{1} \approx 8 \mu \mathrm{F} / \mathrm{cm}^{2}\right)$ obtained from impedance spectra (since capacitance = charge change divided by potential change). As both the long- and short-time values of the $q(t)$ function reveal, the pztc lies somewhere between $-0.3 \mathrm{~V}$ and $-0.1 \mathrm{~V}$.

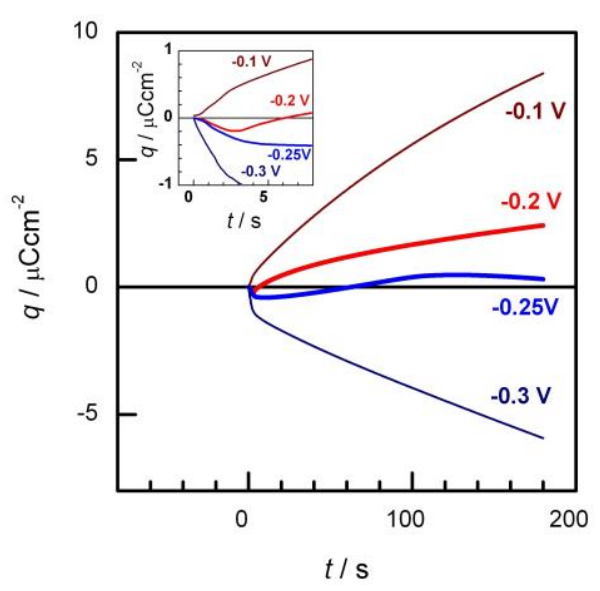

Fig. 7 Integrated immersion current transients at potentials indicated. Inset: the charge in the first seconds. 
(a)

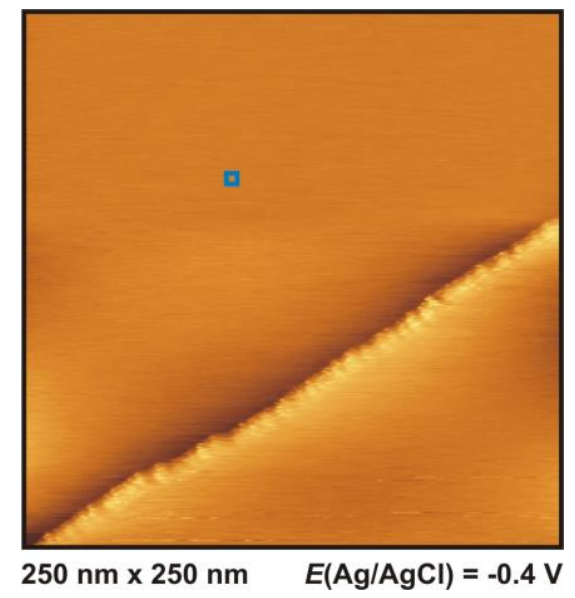

(c)

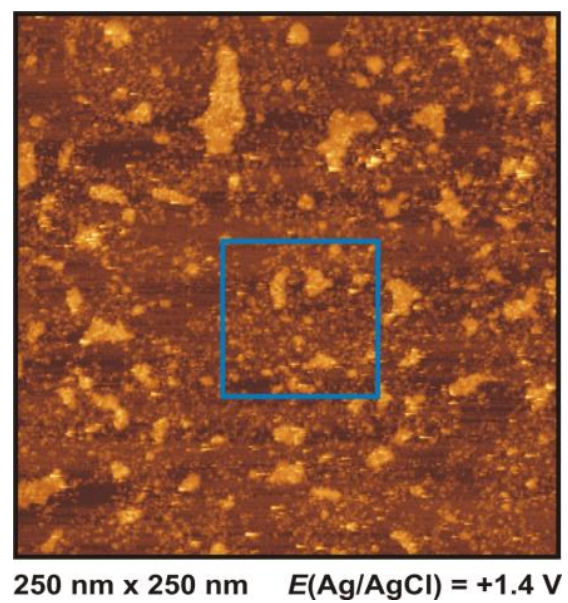

(b)

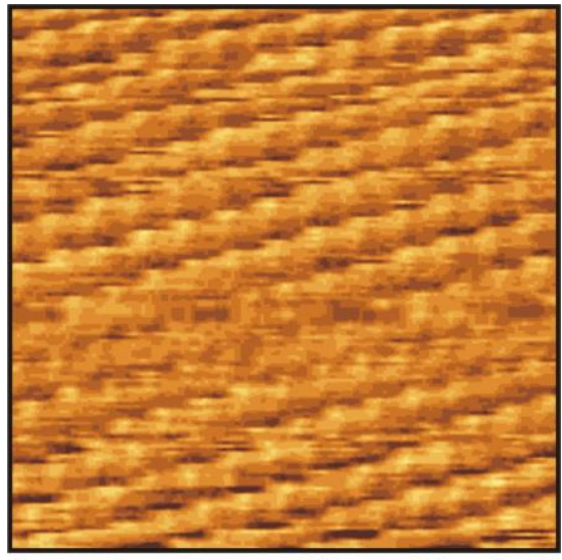

$5 \mathrm{~nm} \times 5 \mathrm{~nm}$

$E(\mathrm{Ag} / \mathrm{AgCl})=-0.4 \mathrm{~V}$

(d)

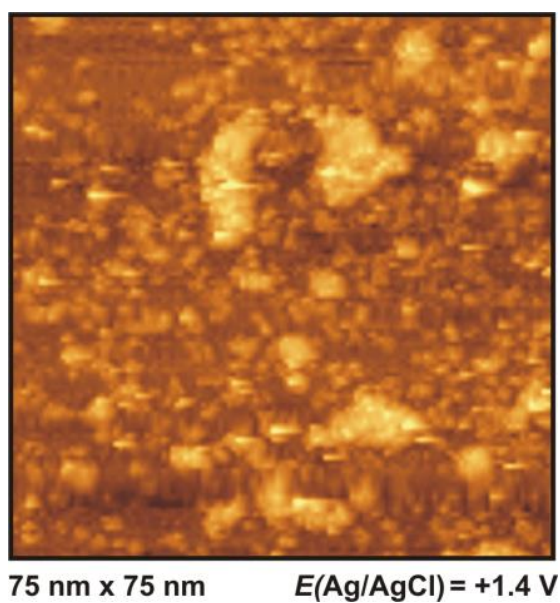

Fig. 8 STM images of HOPG in BMIPF $_{6}$ at potentials and magnifications as indicated. First and second rows: negative and positive to the pztc. The images of the rhs column have been made on the areas marked by squares on Ihs images.

\section{In-situ STM}

In-situ STM studies were accomplished in the stability range of HOPG in the IL. The open circuit potential (approx. $-0.1 \mathrm{~V}$ vs. $\mathrm{Ag} \mid \mathrm{AgCl}$ ) was typically chosen as starting potential. A cathodic potential excursion to $-0.4 \mathrm{~V}$ exhibits large terraces with a step height of $(0.36 \pm 0.05) \mathrm{nm}$ (Fig. 8(a)), which approximately coincides with that of $\mathrm{HOPG}^{38} 0.335 \mathrm{~nm}$.

At this potential the STM imaging is stable and little if any changes of the surface appears for several hours. An enlargement of the terraces shows an ordered adlayer (Fig. 8b) formed by the IL, which is eventually enriched in $\mathrm{BMI}^{+}$cations, since the potential is somewhat more negative than the pztc. Spots are aligned in straight lines, revealing an ordered adlayer of the ionic liquid on HOPG. These spots have a distance of $0.38 \mathrm{~nm}$ to each other, which is larger by a factor of 1.5 compared to a typical in-plane lattice constant of $0.246 \mathrm{~nm}$ for HOPG.

At potentials more positive than $+1.2 \mathrm{~V}$ (i.e., more positive than the location of the spike pair in Fig. 2(a)), the character of the surface immediately changes and nanometer-sized islands of different shapes appear (Figs. 8(c) and 8(d)). Their height is around $0.3 \mathrm{~nm}$ (smaller particles) and $0.6 \mathrm{~nm}$ 
(bigger, brighter particles). The height of the bigger particles is in accordance with the observation of IL "droplets" for this system by AFM. ${ }^{39}$ To understand features at $+1.2 \mathrm{~V}$, we mention the following findings: First, $+1.2 \mathrm{~V}$ is much more positive than the pztc, so anions are closer to the surface than cations. Second, the spikes can be observed neither in $\mathrm{BMITf}_{2} \mathrm{~N}$ nor in a 1:1 mixture of $\mathrm{BMIPF}_{6}$ and $\mathrm{BMITf}_{2} \mathrm{~N}$.

(a)

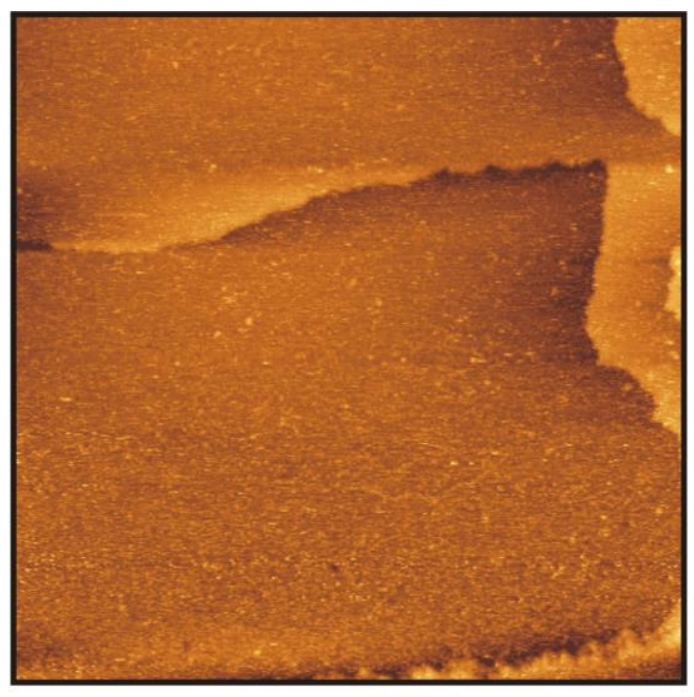

(b)

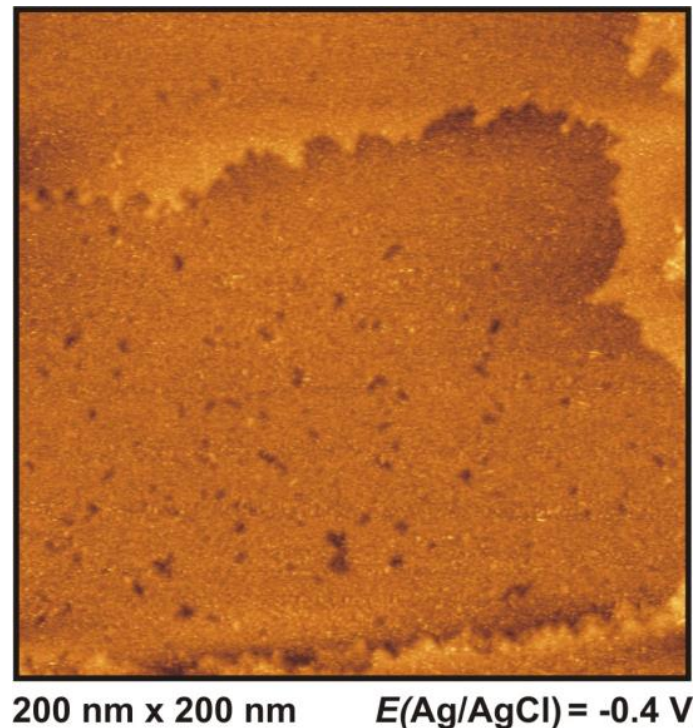

(c)

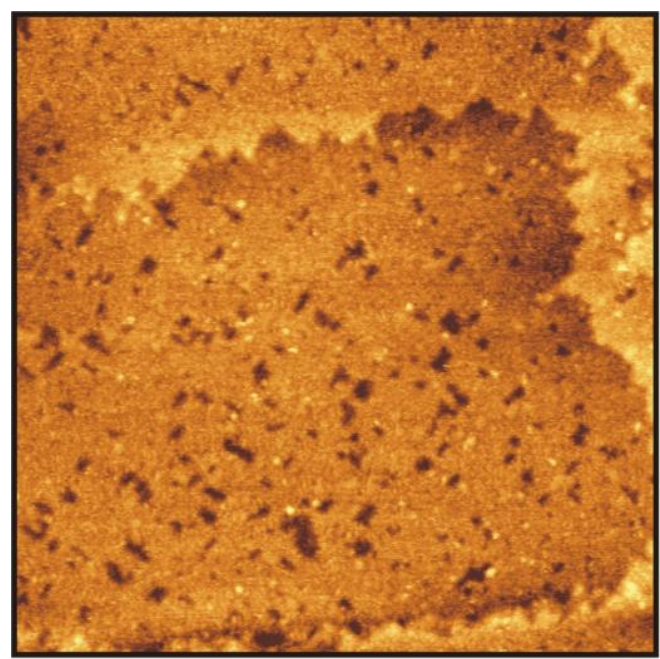

$200 \mathrm{~nm} \times 200 \mathrm{~nm} \quad E(\mathrm{Ag} / \mathrm{AgCl})=-0.4 \mathrm{~V}$

Fig. 9. $\mathrm{Au}(100)$ in $\mathrm{BMIPF}_{6}$ : series of STM images showing the time-dependent formation of holes in the surface. The images (a), (b) and (c) were taken immediately after, 6 minutes after and 30 minutes after placing the electrode to $-0.4 \mathrm{~V}$.

Third, as seen in Fig. $5(\mathrm{~b})$, the $\approx 2 \mu \mathrm{F} / \mathrm{cm}^{2}$ difference of $\operatorname{Re}(C)$ at the two sides of the peak at about $+1.2 \mathrm{~V}$ indicates a significant change of the double layer. Hence, we attribute these findings to some phase transition of a $\mathrm{PF}_{6}$ adlayer.

To demonstrate that $\mathrm{Au}(100)$ is unstable compared to HOPG, the in-situ STM measurement with $\mathrm{Au}(100)$ reported in Ref. 16 has been repeated at a somewhat different potential. Fig. 9(b) of 
Ref. 16 displayed small holes (etch pits), which were formed at $-0.8 \mathrm{~V}$. The evolution of the etchpits is shown in the three images of Fig. 9. At the beginning, a surface with terraces and steps is visible. Within $30 \mathrm{~min}$, the formation of mono-atomic deep pits, $(0.26 \pm 0.05) \mathrm{nm}$, in the terrace could be observed, which are presumably caused by slow etching and dissolution processes of the gold surface in BMIPF $_{6}$ (Figs. 9(b) and 9(c)). A "corrosion" process of similar kind was also reported in previous studies with imidazolium-based ILs ${ }^{16,18,40}$ or pyrrolidinium-based ILs ${ }^{41}$ on $\mathrm{Au}(100)$ and $\mathrm{Au}(111)$, respectively.

\section{Discussion}

\section{Capacitance measurements}

The main finding is the different character of interfacial capacitance spectra of HOPG and Au(100) in $\mathrm{BMIPF}_{6}$. Whereas for Au the spectra exhibit two arcs, in case of HOPG the low frequency arc is missing (Fig.3(a)). The properties of the high frequency arc are rather similar for the two electrodes. For modeling the HOPG interface, the essential branches of the equivalent circuit are those containing $C_{h f}$ and $R_{1}-W_{1}-C_{1}$. For $A u(100)$, however, the branch $R_{2}-W_{2}-C_{2}$ additionally enters the picture. This difference is in accord with the in-situ STM images of the two systems: the HOPG surface is stable (cf. Fig. 8(a) and (b)), whereas on $A u(100)$ a slow rearrangement of the surface proceeds (in a certain potential range, cf. Fig. 9). We attribute the properties of the high frequency arc to the behavior of interfacial IL layers that is common for both systems. The single-arc spectra as those shown in Figs. 4 and 6 and the capacitances plotted in Fig. 5(b) correspond to the double layer of a stable, rearrangement-free conductor in an ionic liquid.

The significance of a single-arc double layer capacitance needs some explanation: Since its introduction, double layer capacity has always been regarded as a quantity which depends on potential, electrode and electrolyte chemical properties, but its independence of frequency has rarely been questioned. In general, there exist three major cases when frequency-dependence of the double layer capacitance appears. First, it may be a consequence of some surface-inhomogeneity; ${ }^{42}$ second, due to some adsorption process of minority components of the electrolyte. ${ }^{43}$ The third case is the appearance of single-arc capacitance spectra in classical, aqueous electrochemical systems, like in Pt-group metals in binary electrolytes ${ }^{44}$; probably the presence is the most obvious in $\mathrm{HCl}$ solutions on $\mathrm{Rh}(111)^{45}$ and $\mathrm{Pt}(100) .{ }^{46}$ In these cases the arcs are associated with $\mathrm{H}^{+}$or $\mathrm{Cl}^{-}$movements between the outer and inner Helmholtz planes.

In the present study a fourth case of frequency dependence shows up: double layer capacitance is a single arc on the complex plane, which signifies some rearrangement of the double layer. The limiting values, $C_{\mathrm{hf}}$ and the $C_{\mathrm{hf}}+C_{1}$ sum are the capacitances in the "frozen" and "relaxed" states of the double layer; the frequency of the topmost point represents a rate of the rearrangement process. This rate exhibits temperature dependence rather similar to that of bulk conductivity, probably due to the similar activation energy of molecular movements at the interface and within the IL bulk. We refrain from giving a molecular level explanation of the rearrangement.

At present, we do not know which of $C_{\mathrm{hf}}$ and $C_{\mathrm{hf}}+\mathrm{C}_{1}$ should be compared with the theoretical predictions. The existing theories ${ }^{19,20,21,22,23,24}$, unfortunately, do not account for frequency dependence, and the predicted values are much larger than any of our $C_{\mathrm{hf}}$ or $C_{\mathrm{hf}}+C_{1}$ values. In addition, we obtain no "bell-shape" or "camel-shape" $C(E)$ curves. Of course, one should keep in mind that due to different experimental methods, cell geometries, chemicals and electrodes in general it is difficult to compare results obtained in different laboratories. ${ }^{11,25,37,47,48,49}$ 
A comment on capacitance measurements with electrodes other than HOPG: Whereas to determine $C_{h f}$ is always possible by taking a high frequency limit of the capacitance, determining the $C_{\mathrm{hf}}+C_{1}$ sum is possible only if the high frequency part can be well-fitted by the equivalent circuit of Fig. 1(a). In most of our studies with $A u(100)$ electrodes, the high frequency arc was always clearly visible on complex-plane plots (cf. Fig. 3 of Ref. 16, Fig. 2b of Ref. 17 or Fig. 4 of Ref. 18). The exceptions are the spectra measured at elevated temperatures (e.g. in Fig. 6 of Ref. 16) where the interfacial processes (as the ones imaged above in Fig. 8) cause the high-frequency arc to be masked by the low-frequency one. Even in this case determination of $C_{\mathrm{hf}}$ is still possible with good accuracy.

Were the interfacial capacitance spectra for HOPG and Au(100) the same at any potential and temperature, we would conclude that the only role of the electrode is to provide the electric field upon the double layer. As the data displayed in Fig. 5(a) show, some small differences of the spectra exist, thus there are also other-than-electrostatic ("chemical") interactions between the electrodes and the IL.

In-situ STM

In general, the STM images of HOPG in ILs are of lower quality than those obtained in air or in aqueous solutions. This is usually attributed to a strong binding of the IL ions to HOPG and to an eventual thermal drift of the tip. ${ }^{50}$ These effects may hinder the reliable determination of structure geometries. Bearing in mind the above considerations, the periodicity seen in the micrograph of Fig. 8(b), recorded at potentials somewhat negative to the pztc, has two possible explanations. It may be attributed to a distorted HOPG surface, but also to an ordered, cation-rich adlayer. In the latter case the characteristic length - that is about 1.5 times larger than the lattice constant of HOPG - implies a more dense packing than that predicted by MD simulations. ${ }^{10}$ In Ref. [10], a defective 2D-hexagonal lattice of the adsorbed ions with a lattice spacing approximately four times larger than that of HOPG is determined. The discrepancy between the theoretical predictions of [10] and our experimental observations is a current research task in our institute and new simulation models are intended to be developed using our distinguished parameters. The appearance of patches has already been reported in Ref. 51 as the formation of thin films, nanometer-sized "droplets" and "drop-on-layer" structures of this ionic liquid. Films which cover the surface with a thickness up to $2 \mathrm{~nm}$ - corresponding to 4 IL layers - seem to be solid and indicate that IL molecules are organized into various micro-morphologies at room-temperature. ${ }^{51}$ Theoretical studies confirmed the existence of spatial heterogeneities at the interface due to the formation of polar and non-polar domains of the IL-molecules, as well as the existence of highly compressible alternating multilayers. ${ }^{52,53,54,55,56}$

At very high negative potentials we could not find ordered superstructures such as those exist on $\mathrm{Au}(100)$ in $\mathrm{BMIPF}_{6}$, where ordered double-row structures of the cations could be observed (Fig. 9. of Ref. 16). Ordering could be detected on HOPG in $\mathrm{OMITf}_{2} \mathrm{~N}$ at very negative potentials in a combined STM, AFM and DFT study ${ }^{50}$. However, at these potentials, apparently, the HOPG and the BMI cations do not fit to each other and the double-row ordering of the cations is not possible for this electrode-cation combination.

At $+1.4 \mathrm{~V}$, that is positive of the characteristic CV spikes, formation of islands is observed (Figs. 8(c) and $8(\mathrm{~d})$ ). The height is around $0.3 \mathrm{~nm}$ and $0.6 \mathrm{~nm}$ for the smaller and larger islands, respectively, implying probably mono- and bilayer-high islands. Taking also into account that the sharp peaks appear only in pure $\mathrm{BMIPF}_{6}$ - i.e. the $\mathrm{CV}$ spikes mark some phase transition of the $\mathrm{PF}_{6}{ }^{-}$adlayer these islands are probably $\mathrm{PF}_{6}{ }^{-}$rich domains. Another confirmation of this assumption is the fact 
that the molecule size of this octahedron-sized ion is around $0.32 \mathrm{~nm}$, which implies probably mono- and bilayer-high islands.

The connection of slow capacitive processes with surface rearrangement processes of single crystalline $\mathrm{Au}$ in ILs was put forward by Drüschler and coworkers recently. ${ }^{49}$ They reported fast, slow and an ultra-slow sub- $\mathrm{Hz}$ capacitive processes for $\mathrm{Au}(111)$ in 1-butyl-1-methylpyrrolidiniumtris(pentafluoroethyl)trifluorophosphate ( $\mathrm{Py}_{1,4} \mathrm{FAP}$ ). The ultra-slow capacitive contribution was assumed to be related to a vacancy formation in the surface. This assumption is in line with our present study: their "ultra-slow" capacitive process corresponds to our low frequency arc; the vacancy formation is a similar surface rearrangement process visualized by the in-situ STM image series of

Fig. 9. We note that pit formation has also been reported for $\mathrm{Au}(100)$ in $\mathrm{BMIBF}_{4}{ }^{40}$

\section{Conclusions}

The HOPG | $\mathrm{BMIPF}_{6}$ interface has been studied in correspondence to our previous investigation of $\mathrm{Au}(100) \mid \mathrm{BMIPF}_{6}{ }^{16}$ The main observation is the similarity of the high-frequency part of the capacitance spectra on the two systems. In contrast, the capacitance behavior at low frequencies is markedly different, which is attributed to the different stability of the two surfaces. As in-situ STM images reveal, the HOPG surface is stable, whereas the $A u(100)$ surface is continuously rearranged; step edges move, etch pits are formed, the initially uniform crystal face corrodes. These "corrosion" processes modify the low-frequency impedance/capacitance: a resistive-capacitive branch (empirically $\mathrm{R}_{2}-\mathrm{W}_{2}-\mathrm{C}_{2}$ ) parallel to the double layer capacitance elements appears in the equivalent circuit of Fig. 1.

From a double-layer capacity point of view, the $C_{h f}||\left(R_{1}-W_{1}-C_{1}\right)$ elements are the main terms of the equivalent circuit being dominant at high frequencies of the capacitance spectra. Since the arc's parameters (including the temperature dependences) are similar for HOPG and Au(100), we attribute this arc to the interfacial layer of the IL. Since $C_{\mathrm{hf}}$ and $C_{\mathrm{hf}}+C_{1}$ are the limiting values of capacitance at high and low frequency, respectively, we regard these two quantities as double-layer capacitances characterizing the "frozen" and "relaxed" states. As far as we know, at the moment no metal | IL double layer theory exists by which $C_{\mathrm{hf}}$, and $C_{\mathrm{hf}}+C_{1}$ could be interpreted.

Whereas the HOPG is stable under IL, in-situ STM images reveal different IL structures at different potentials. At potentials somewhat negative of the pztc, an ordered structure on the surface could be observed, which has a 1.5 times larger lattice constant than that of HOPG. At +1.4V, that is, positive of characteristic CV spikes - marking probably some phase transition of the $\mathrm{PF}_{6}{ }^{-}$adlayer formation of islands occurs.

\section{Acknowledgments}

This cooperation was made possible by the exchange project of the Deutscher Akademischer Austauschdienst and the Hungarian Fellowship Board, No. 39706. The support of Hungarian funding agency OTKA (under contract No. 112034) is highly appreciated. 


\section{References}

1 M. Freemantle, Introduction to lonic Liquids, RSC Publishing, 2009.

2 B. Kirchner, in Topics of Current Chemistry, ed. B. Kirchner, Springer, 2010, vol. 290.

3 A. Kokorin, Ionic Liquids: Application and Perspectives, InTech, 2011.

4 Ionic Liquids in Synthesis, eds. P. Wasserscheid and T. Welton, Wiley, 2008, vol. 1.

5 A. Balducci, R. Dugas, P. L. Taberna, P. Simon, D. Plée, M. Mastragostino and S. Passerini, J. Power Sources, 2007, $165,922$.

6 T. Tamura, T. Hachida, K. Yoshida, N. Tachikawa, K. Dokko and M. Watanabe, J. Power Sources, 2010, 195, 6095.

7 A. Lewandowski and A. Świderska-Mocek, J. Power Sources, 2009, 194, 601.

8 J. S. Wilkes and M. J. Zaworotko, J. Chem. Soc. Chem. Commun., 1992, 13, 965.

9 F. Endres, D. MacFarlane and A. Abbott, Electrodeposition from Ionic Liquids, Wiley, 2008, ch. 11.

10 S. Kislenko, I. S. Samoylov and R. H. Amirov, Phys. Chem. Chem. Phys., 2009, 11, 5584.

11 M. Drüschler, B. Huber, S. Passerini and B. Roling, J. Phys. Chem. C, 2010, 114, 3614.

12 R. Hayes, Sh. Zein El Abedin and R. Atkin, J. Phys. Chem. B, 2009, 113, 7049.

13 S. Perkin, L. Crowhurst, H. Niedermeyer, T. Welton, A. M. Smith and N. N. Gosvami, Chem. Commun., 2011, 47, 6572.

14 M. Gnahm, T. Pajkossy and D. M. Kolb, Electrochim. Acta, 2010, 55, 6212.

15 T. Pajkossy and D. M. Kolb, Electrochem. Comm., 2011, 13, 284.

16 M. Gnahm, C. Müller, R. Répánszki, T. Pajkossy and D. M. Kolb, Phys. Chem. Chem. Phys., 2011, 13, 11627.

17 M. Gnahm, C. Berger, M. Arkhipova, H. Kunkel, T. Pajkossy, G. Maas and D. M. Kolb, Phys.Chem.Chem.Phys., 2012, 14, 10647.

18 C. Müller, S. Vesztergom, T. Pajkossy and T. Jacob, J. Electroanal. Chem., 2015, 737, 218.

19 A. A. Kornyshev, J. Phys. Chem. B, 2007, 111, 5545.

20 M. V. Fedorov and A. A. Kornyshev, Electrochim. Acta, 2008, 53, 6835.

21 N. Georgi, A. A. Kornyshev and M. V. Fedorov, J. Electroanal. Chem., 2010, 649, 261.

22 M. V. Fedorov, N. Georgi and A. A. Kornyshev, Electrochem. Commun., 2010, 12, 296.

23 A. A. Kornyshev, N. B. Luque and W. Schmickler, J. Solid State Electrochem., 2014, 18, 1345.

24 K. B. Oldham, J. Electroanal. Chem., 2008, 613, 131.

25 V. Lockett, M. Horne, R. Sedev, T. Rodopoulos and J. Ralston, Phys. Chem. Chem. Phys., 2010, 12, 12499.

26 A. Noda, K. Hayamizu and M. Watanabe, J. Phys. Chem. B, 2001, 105, 4603.

27 H. Tokuda, K. Hayamizu, K. Ishii, Md. A. bin H. Susan and M. Watanabe J. Phys. Chem. B, 2004, $108,16593$.

28 H. Tokuda, K. Hayamizu, K. Ishii, Md. A. bin H. Susan and M. Watanabe, J. Phys. Chem. B, 2005, 109, 6103.

29 H. Tokuda, K. Ishii, Md. A. bin H. Susan, S. Tsuzuki, K. Hayamizu and M. Watanabe, J. Phys. Chem. B, 2006, 110, 2833.

30 N. Bodappa, P. Broekmann, Y.-Ch. Fu, J. Furrer, Y. Furue, T. Sagara, H. Siegenthaler, H. Tahara, S. Vesztergom, K. Zick and Thomas Wandlowski, J. Phys. Chem. C, 2015, 119, 1067.

31 W. Lu, P. Soukiassian and J. Boeckl, MRS Bull., 2012, 37, 1119.

32 M. Gnahm, and D. M. Kolb, J. Electroanal. Chem., 2011, 651, 250.

33 C. Müller, S. Vesztergom, T. Pajkossy and T. Jacob, Electrochim. Acta, 2016, 188, 512. 
34 W. Ehm, H. Göhr, R. Kaus, B. Röseler, C. A. Schiller, ACH-Models in chemistry, 2000, 137, 145.

35 B. A. Boukamp, Solid State lonics, 1986, 20, 31.

36 T. Pajkossy, Electrochim. Acta, 2010, 56, 7246.

37 F. Silva, C. Gomes, M. Figueiredo, R. Costa, A. Martins and C. M. Pereira, J. Electroanal. Chem., 2008, $622,153$.

38 Y. Yokota, T. Harada and K. Fukui, Chem. Commun., 2010, 46, 8627.

39 F.-C. Zhang, M.-L. Sha, X.-P. Ren, G. Z. Wu, J. Hu and Y. Zhang, Chin. Phys. Lett., 2010, 27, 086101.

40 Y. Z. Su, Y.-Ch. Fu, J. W. Yon, Z. B. Chen and B.-W. Mao, Angew. Chemie Int. Ed., 2009, 48, 5148.

41 R. Atkin, Sh. Zein El Abedin, R. Hayes, L. H. S. Gasparotta, N. Borisenko, F. Endres, J. Phys. Chem. C, 2009, 113, 13266.

42 T. Pajkossy, Solid State lonics, 2005, 176, 1997.

43 Z. Kerner, T. Pajkossy, Electrochim. Acta, 2002, 47, 2055.

44 T. Pajkossy and D. M. Kolb, Electrochim. Acta, 2008, 53, 7403.

45 T. Pajkossy and D. M. Kolb, Electrochim. Acta, 2009, 54, 3594.

46 T. Pajkossy, Z. Phys. Chem., 2012, 226, 935.

47 M. M. Islam, M. T. Alam, T. Okajima and T. Ohsaka, J. Phys. Chem. C, 2009, 113, 3386.

48 C. Cannes, H. Cachet, C. Debiemme-Chouvy, C. Deslouis, J. de Sanoit, C. Le Naour, V. A. Zinovyeva, J. Phys. Chem. C, 2013, 117, 22915.

49 M. Drüschler, N. Borisenko, J. Wallauer, C. Winter, B. Huber, F. Endres, B. Roling, Phys. Chem. Chem. Phys., 2012, 14, 5090.

50 T. Carstens, R. Gustus, O. Höfft, N. Borisenko, F. Endres, H. Li, R. J. Wood, A. J. Page and R. Atkin, J. Phys. Chem. C, 2014, 118, 10833.

51 Z. Fu-Chun, S. Mao-Lin, R. Xiu-Ping, W. Guo-Zhong, H. Jun and Z. Yi, Chin. Phys. Lett., 2010, $27,86101$.

52 S. Wang, S. Li, Z. Cao and T. Yan, J. Phys. Chem. C, 2010, 114, 990.

53 S. Maolin, Z. Fuchun, W. Guozhong, F. Haiping, W. Chunlei, C. Shimou, Z. Yi, and H. Jun, J. Chem. Phys., $2008,128,1$. 54 Z. Hu, J. Vatamanu, O. Borodin and D. Bedrov, Phys. Chem. Chem. Phys., 2013, 15, 14234.

55 J. Vatamanu, O. Borodin and G. D. Smith, J. Am. Chem. Soc., 2010, 132, 14825.

56 J. Vatamanu, O. Borodin, D. Bedrov and G. D. Smith, J. Phys. Chem. C, 2012, 116, 7940. 